ISSN = 1980-993X - doi:10.4136/1980-993X
www.ambi-agua.net
E-mail: ambi-agua@agro.unitau.br
Tel.: (12) 3625-4212

\title{
The potential of Zea mays, Commelina bengelensis, Helianthus annuus and Amaranthus hybridus for phytoremediation of waste water
}

(http://dx.doi.org/10.4136/ambi-agua.684)

\author{
Oyaro Nathan'; Kimenyu Phylis Njeri²; Okong'o Eric Rang'ondi \\ Chacha Joseph Sarima ${ }^{1}$ \\ ${ }^{1}$ Narok university College, Department of chemistry, Box 861-20500, Narok, Kenya, \\ e-mails: oyarokema@yahoo.com; chachajs@yahoo.com, \\ ${ }^{2}$ Jomo Kenyatta University of Agriculture \& Technology, Department of chemistry, \\ Box 62000-00200, Nairobi, Kenya, Tel. 254 6752223, Fax 254 6752197, \\ e-mails: kimenyupn2012@yahoo.com; erickokongo67@yahoo.com
}

\begin{abstract}
Waste-water from domestic use and from industrial effluent burden the water systems with high levels of heavy metal hence there is need to remove these heavy metals so that the waste water can be recycled for use for household or irrigation. The present study has screened Zea mays (maize), Commelina bengelensis (wondering jew), Helianthus annuus (sunflower) and Amaranthus hybridus (amaranthus) for their ability to bioaccumulate $\mathrm{Pb}, \mathrm{Cu}$, $\mathrm{Cd}$ and $\mathrm{Zn}$ metals. The results obtained show that the $H$. annuus and $C$. bengelensis plant have promising potential for removal of $\mathrm{Pb}, \mathrm{Cu}$ and $\mathrm{Cd}$ from wastewater though their ability to remove $\mathrm{Zn}$ from contaminated solutions is not much different from that of $Z$. mays and $A$. hybridus.
\end{abstract}

Keywords: AAS, lead, copper, zinc, cadmium.

\section{O potencial de Zea mays, Commelina bengelensis, Helianthus annuus $e$ Amaranthus hybridus para fitorremediação de águas residuárias}

\section{RESUMO}

Nas águas residuárias de origem doméstica ou esgoto sanitário, níveis elevados de metais tóxicos poluem os sistemas hídricos e, por conseguinte, há necessidade de eliminar esses elementos para que as águas residuárias possam ser recicladas para uso doméstico ou irrigação. O presente estudo avaliou a capacidade das espécies Zea mays (milho), Commelina bengelensis (trapoeraba), Helianthus annuus (girassol) e Amaranthus hybridus (amaranto) para bioacumulação de $\mathrm{Pb}, \mathrm{Cu}, \mathrm{Cd}$ e $\mathrm{Zn}$. Os resultados revelaram que o $H$. annuus e a $C$. bengelensis têm potencial para remoção do $\mathrm{Pb}, \mathrm{Cu}$ e $\mathrm{Cd}$ de águas residuárias, entretanto, a capacidade delas para remoção de $Z n$ não é muito diferente das espécies $Z$. mays e $A$. hybridus.

Palavras-chave: AAS, chumbo, cobre, zinco, cádmio.

\section{INTRODUCTION}

Heavy metals are major pollutants in marine, ground, industrial and even treated wastewater (Valdman et al., 2001). The heavy metals may come from natural sources, leached from rocks and soils according to their geochemical mobility or come from anthropogenic 
NATHAN, O.; NJERI, K. P.; RANG'ondi, O. E.; SARIMA, C. J. The potential of Zea mays, Commelina bengelensis, Helianthus annuus and Amaranthus hybridus for phytoremediation of waste water. Ambi-Agua, Taubaté, v. 7, n. 3, p. 51-60, 2012. (http://dx.doi.org/10.4136/ambi-agua.684)

sources, as a result of human land occupation and industrial pollution (Espinoza-Quiñones et al., 2005).

Industrial waste constitutes the major source of various kinds of metal pollution in natural waters. The important toxic metals $\mathrm{Cd}, \mathrm{Zn}, \mathrm{Ni}$ and $\mathrm{Pb}$ find their way to the water bodies through waste waters Ajmal et al. (1998). Due to their non- biodegradability and persistence, heavy metals can accumulate in the environment such as in food chains and thus may pose a significant danger to human health (Bakkaloglu et al., 1998).

Rhizofil tration, the use of plants both terrestrial and aquatic to absorb, concentrate and precipitate contaminants from polluted aqueous sources with low contaminant concentration in their roots, can partially be used to treat industrial discharge. It can be used for $\mathrm{Pb}, \mathrm{Cd}, \mathrm{Cu}$, $\mathrm{Ni}, \mathrm{Zn}$ and $\mathrm{Cr}$, which are primarily retained within roots (Chaudhry et al., 1998). For example plants like sunflower, Indian mustard, tobacco, rye, spinach and corn have been studied for their ability to remove $\mathrm{Pb}$ from effluent, with sunflower having the greatest ability (Ghosh and Singh, 2005). Indian mustard has proven to be effective in removing a wide concentration range of lead (4-500 $\mathrm{mg} \mathrm{L}^{-1}$ ) (Raskin and Ensley, 2000).

The technology has been tested in the field with uranium-contaminated water at concentrations of 21-874 $\mu \mathrm{g} \mathrm{L}^{-1}$; the treated uranium concentration reported by Dushenkov et al. (1995) was $<20 \mu \mathrm{g} \mathrm{L}^{-1}$ before discharge into the environment.

This plant-assisted bioremediation is most effective if groundwater is within 10 feet of the surface (Cunningham et al., 1997) and is applicable to sites with large volumes of groundwater with low levels of contamination that have to be cleaned to low (strict) standards (Salt et al., 1997).

In this study, the efficiency of Helianthus annuus (sunflower), Commelina bengelensis (wondering Jew), Zea mays (maize) and Amaranthus hybridus (amaranth) has been studied in the process of heavy metal removing from both single metal solutions and various mixtures. Metals of interest were $\mathrm{Cd}, \mathrm{Pb}, \mathrm{Cu}$ and $\mathrm{Zn}$ based on their industrial applications and potential pollution impact on the environment. $\mathrm{Cd}$ and $\mathrm{Pb}$ are cumulative poisons, highly toxic to humans, plant and animals (Low et al., 2000). Cu and $\mathrm{Zn}$ play major roles in modern industry and in the vicinity of extraction or processing plants, the emissions arising are certainly capable of causing an undesirable contamination of agricultural products and therefore recommended not to omit these metals from scrutiny.

\section{MATERIALS AND METHODS}

This study was conducted in a greenhouse as pot experiments on distilled water at Jomo Kenyatta University of Agriculture and Technology in Kenya. The experiment was carried out during 2008 - 2011 period. The experimental layout for single metal solutions comprised 8 treatments, a control with no metal added, and 7 treatments for which the dose of heavy metal was increasingly higher (Table 1). Heavy metals were added as salts of $\mathrm{Pb}\left(\mathrm{NO}_{3}\right)_{2}$, $\mathrm{Cu}\left(\mathrm{NO}_{3}\right)_{2} 5 \mathrm{H}_{2} \mathrm{O}, \mathrm{Zn}\left(\mathrm{NO}_{3}\right)_{2} .7 \mathrm{H}_{2} \mathrm{O}$ and $\mathrm{Cd}\left(\mathrm{NO}_{3}\right)_{2} \cdot 4 \mathrm{H}_{2} \mathrm{O}$. The experiment was carried out in triplicate and stratified sampling was done to identify the pot but at each pot random sampling was employed in choosing the plant for analysis.

To compare the uptake of these metals from single solutions (uncombined state) and uptake from mixture solutions (combined state), the mixture solutions were prepared by dissolving the salts of the heavy metals in distilled water in order to achieve the same concentrations as those of single solutions as per Table 1 .

Zea mays and Helianthus annuus were germinated on a tray in the green house for 14 days while Amaranthus hybridus seedlings and Commelina bengelensis cuttings were obtained from the university farm, transplanted on tray in the greenhouse for the same number of days. The plants were uprooted and three seedlings per pot were suspended in the 
NATHAN, O.; NJERI, K. P.; RANG'ondi, O. E.; SARIMA, C. J. The potential of Zea mays, Commelina bengelensis, Helianthus annuus and Amaranthus hybridus for phytoremediation of waste water. Ambi-Agua, Taubaté, v. 7, n. 3, p. 51-60, 2012. (http://dx.doi.org/10.4136/ambi-agua.684)

contaminated solutions with the roots completely immersed. Every four days for twelve days, one plant was removed from each pot, put in polyethylene bag immediately sealed and transported to the laboratory for analysis.

Table 1. Dose of heavy metals $\left(\mathrm{mg} \mathrm{L}^{-1}\right)$ added to distilled water for treatments 0 - VII.

\begin{tabular}{c|cccc}
\hline Treatment & Cd & Cu & Zn & Pb \\
\hline Control & 0 & 0 & 0 & 0 \\
I & 10 & 20 & 50 & 30 \\
II & 20 & 40 & 100 & 60 \\
III & 40 & 80 & 200 & 120 \\
IV & 80 & 160 & 400 & 240 \\
V & 160 & 320 & 800 & 480 \\
VI & 320 & 640 & 1600 & 960 \\
VII & 640 & 1280 & 3200 & 1920 \\
\hline
\end{tabular}

In the laboratory, samples were washed with distilled water, bench dried for 5 days then oven dried at $105^{\circ} \mathrm{C}$ for 12 hours and each separately ground to a powder in a porcelain mortar. The powder was then stored in plastic bottles ready for analysis.

The digestion of samples was carried out as follows: $0.1 \mathrm{~g}$ of the oven-dried powder was put in a $100 \mathrm{~mL}$ conical flask and $5 \mathrm{~mL}$ of the tri-acid mixture (nitric, perchloric and sulfuric) in the ratio 3:1:1 was added and let to digest on a hot plate until brown fumes disappeared and white fumes observed. The samples were then cooled, diluted with a $4 \%$ nitric acid and filtered into $100 \mathrm{~mL}$ volumetric flasks. These were then made up to the mark with distilled water and analyzed using Atomic Absorption Spectrophotometer (AAS).

\section{RESULTS AND DISCUSSION}

Table 2 presents the $\mathrm{Pb}$ concentrations in the A. hybridus, Z. mays, C. bengelensis and $H$. annuus were in the range of (0.061-1.211), (0.046-3.921), (1.354-7.632) and (1.168-8.558) $\mathrm{g}$ $\mathrm{kg}^{-1}$, respectively.

These different values show that the uptake of $\mathrm{Pb}$ is plant dependent and also depends on the concentration of $\mathrm{Pb}$ in solution. The $\mathrm{Pb}$ concentration in $C$. bengelensis and $H$. annuus were above the value used to define hyper accumulation $\left(1.0 \mathrm{~g} \mathrm{~kg}^{-1}\right)$, hence these plants in solutions can be referred to as hyperaccumulators of $\mathrm{Pb}$. These values are comparable to values of $0.009-4.561 \mathrm{~g} \mathrm{~kg}^{-1}$ obtained by Lombi et al. (2001) in roots of Z. mays grown in soils with EDTA. In another research by Podar et al. (2004), the concentration of $\mathrm{Pb}$ ranged from $0.001-0.030 \mathrm{~g} \mathrm{~kg}^{-1}$ in $H$. annuus and $0.000-0.018 \mathrm{~g} \mathrm{~kg}^{-1}$ in Z. mays grown in soil contaminated with mine spoils. These values are lower than those obtained in this study. The results obtained in the current study are higher than those of Carlson et al. (1975), who found that the amount of $\mathrm{Pb}$ in leaves of $\mathrm{H}$. annuus and $Z$. mays treated with up to $500 \mathrm{mg} / \mathrm{lof} \mathrm{Pb}$, as $\mathrm{PbCl}_{2}$, averaged $0.004 \mathrm{~g} \mathrm{~kg}^{-1} \mathrm{~Pb}$ and was not statistically different from the $\mathrm{Pb}$ concentration in the control (untreated) plants.

No toxicity was observed for the four plants for all the treatments under investigation. This observation is in agreement with findings of Vyslouzilova et al. (2003) who found out that willow plants grew without any physiological symptoms of phytotoxicity in soil supplied with $2.000 \mathrm{~g} \mathrm{~kg}^{-1}$. The lack of $\mathrm{Pb}$ toxicity is probably due to its immobilization in roots as reported by Vyslouzivola et al. (2003) and Stoltz and Greger (2002). 
NATHAN, O.; NJERI, K. P.; RANG'ondi, O. E.; SARIMA, C. J. The potential of Zea mays, Commelina bengelensis, Helianthus annuus and Amaranthus hybridus for phytoremediation of waste water. Ambi-Agua, Taubaté, v. 7, n. 3, p. 51-60, 2012. (http://dx.doi.org/10.4136/ambi-agua.684)

Table 2. Concentration of lead $\left(\mathrm{g} \mathrm{kg}^{-1}\right)$ in Z. mays, C. bengelensis and A. hybridus grown in contaminated soil for the first, second and third harvests over three months period.

\begin{tabular}{|c|c|c|c|c|c|c|c|c|c|c|c|c|}
\hline $\begin{array}{c}\text { Metal } \\
\mathbf{P b}\end{array}$ & \multicolumn{4}{|c|}{$\mathbf{1}^{\text {st }}$ (Month) } & \multicolumn{4}{|c|}{$2^{\text {nd }}$ (Month) } & \multicolumn{4}{|c|}{$3^{\text {rd }}$ (Month) } \\
\hline Treat. & $\begin{array}{c}\text { A. } \\
\text { hybridus }\end{array}$ & $\begin{array}{c}Z . \\
\text { mays }\end{array}$ & $\begin{array}{c}\boldsymbol{C} . \\
\text { bengelensis }\end{array}$ & $\begin{array}{c}\boldsymbol{H} . \\
\text { annuus }\end{array}$ & $\begin{array}{c}A . \\
\text { hybridus }\end{array}$ & $\begin{array}{c}\boldsymbol{Z} . \\
\text { mays }\end{array}$ & $\begin{array}{c}C . \\
\text { bengelensis }\end{array}$ & $\begin{array}{c}\boldsymbol{H} . \\
\text { annuus }\end{array}$ & $\begin{array}{c}A . \\
\text { hybridus }\end{array}$ & $\begin{array}{l}\boldsymbol{Z} . \\
\text { mays }\end{array}$ & $\begin{array}{c}\boldsymbol{C} . \\
\text { bengelensis }\end{array}$ & $\begin{array}{c}\boldsymbol{H} . \\
\text { annuus }\end{array}$ \\
\hline 0 & $\begin{array}{c}0.000 \\
\pm \\
0.000\end{array}$ & $\begin{array}{c}0.000 \\
\pm \\
0.000\end{array}$ & $\begin{array}{c}0.000 \\
\pm \\
0.000\end{array}$ & $\begin{array}{c}0.000 \\
\pm \\
0.000\end{array}$ & $\begin{array}{c}0.000 \\
\pm \\
0.000\end{array}$ & $\begin{array}{c}0.000 \\
\pm \\
0.000\end{array}$ & $\begin{array}{c}0.000 \\
\pm \\
0.000\end{array}$ & $\begin{array}{c}0.000 \\
\pm \\
0.000\end{array}$ & $\begin{array}{c}0.000 \\
\pm \\
0.000\end{array}$ & $\begin{array}{c}0.000 \\
\pm \\
0.000\end{array}$ & $\begin{array}{c}0.000 \\
\pm \\
0.000\end{array}$ & $\begin{array}{c}0.000 \\
\pm \\
0.000\end{array}$ \\
\hline 30 & $\begin{array}{c}0.272 \\
\pm \\
0.039\end{array}$ & $\begin{array}{c}0.046 \\
\pm \\
0.016\end{array}$ & $\begin{array}{c}1.535 \\
\pm \\
0.030\end{array}$ & $\begin{array}{c}1.702 \\
\pm \\
0.014\end{array}$ & $\begin{array}{c}0.167 \\
\pm \\
0.040\end{array}$ & $\begin{array}{c}0.061 \\
\pm \\
0.015\end{array}$ & $\begin{array}{c}1.607 \\
\pm \\
0.037\end{array}$ & $\begin{array}{c}4.250 \\
\pm \\
0.053\end{array}$ & $\begin{array}{c}0.061 \\
\pm \\
0.0305\end{array}$ & $\begin{array}{c}0.101 \\
\pm \\
0.023\end{array}$ & $\begin{array}{c}1.354 \\
\pm \\
0.066\end{array}$ & $\begin{array}{c}4.773 \\
\pm \\
0.045\end{array}$ \\
\hline 60 & $\begin{array}{c}0.231 \\
\pm \\
0.046\end{array}$ & $\begin{array}{c}0.096 \\
\pm \\
0.049\end{array}$ & $\begin{array}{c}1.842 \\
\pm \\
0.051\end{array}$ & $\begin{array}{c}2.767 \\
\pm \\
0.173\end{array}$ & $\begin{array}{c}0.070 \\
\pm \\
0.048\end{array}$ & $\begin{array}{c}0.151 \\
\pm \\
0.027\end{array}$ & $\begin{array}{c}3.293 \\
\pm \\
0.067\end{array}$ & $\begin{array}{c}4.038 \\
\pm \\
0.045\end{array}$ & $\begin{array}{c}0.117 \\
\pm \\
0.018\end{array}$ & $\begin{array}{c}0.222 \\
\pm \\
0.077\end{array}$ & $\begin{array}{c}1.851 \\
\pm \\
0.054\end{array}$ & $\begin{array}{c}5.634 \\
\pm \\
0.046\end{array}$ \\
\hline 120 & $\begin{array}{c}0.222 \\
\pm \\
0.053\end{array}$ & $\begin{array}{c}0.237 \\
\pm \\
0.026\end{array}$ & $\begin{array}{c}2.067 \\
\pm \\
0.018\end{array}$ & $\begin{array}{c}2.067 \\
\pm \\
0.020\end{array}$ & $\begin{array}{c}0.206 \\
\pm \\
0.038\end{array}$ & $\begin{array}{c}0.174 \\
\pm \\
0.050\end{array}$ & $\begin{array}{c}3.456 \\
\pm \\
0.067\end{array}$ & $\begin{array}{c}5.614 \\
\pm \\
0.074\end{array}$ & $\begin{array}{c}0.1917 \\
\pm \\
0.035\end{array}$ & $\begin{array}{c}0.202 \\
\pm \\
0.038\end{array}$ & $\begin{array}{c}2.712 \\
\pm \\
0.093\end{array}$ & $\begin{array}{c}4.865 \\
\pm \\
0.028\end{array}$ \\
\hline 240 & $\begin{array}{c}0.634 \\
\pm \\
0.097\end{array}$ & $\begin{array}{c}0.671 \\
\pm \\
0.046\end{array}$ & $\begin{array}{c}4.321 \\
\pm \\
0.029\end{array}$ & $\begin{array}{c}1.168 \\
\pm \\
0.046\end{array}$ & $\begin{array}{c}0.530 \\
\pm \\
0.046\end{array}$ & $\begin{array}{c}0.771 \\
\pm \\
0.026\end{array}$ & $\begin{array}{c}3.932 \\
\pm \\
0.098\end{array}$ & $\begin{array}{c}4.815 \\
\pm \\
0.010\end{array}$ & $\begin{array}{c}0.827 \\
\pm \\
0.114\end{array}$ & $\begin{array}{c}0.630 \\
\pm \\
0.109\end{array}$ & $\begin{array}{c}3.867 \\
\pm \\
0.022\end{array}$ & $\begin{array}{c}5.084 \\
\pm \\
0.028\end{array}$ \\
\hline 480 & $\begin{array}{c}0.817 \\
\pm \\
0.066\end{array}$ & $\begin{array}{c}0.671 \\
\pm \\
0.023\end{array}$ & $\begin{array}{c}7.236 \\
\pm \\
0.037\end{array}$ & $\begin{array}{c}1.461 \\
\pm \\
0.019\end{array}$ & $\begin{array}{c}0.530 \\
\pm \\
0.031\end{array}$ & $\begin{array}{c}1.295 \\
\pm \\
0.023\end{array}$ & $\begin{array}{c}7.632 \\
\pm \\
0.048\end{array}$ & $\begin{array}{c}4.460 \\
\pm \\
0.038\end{array}$ & $\begin{array}{c}0.832 \\
\pm \\
0.085\end{array}$ & $\begin{array}{c}0.913 \\
\pm \\
0.068\end{array}$ & $\begin{array}{c}5.029 \\
\pm \\
0.148\end{array}$ & $\begin{array}{c}5.360 \\
\pm \\
0.016\end{array}$ \\
\hline 960 & $\begin{array}{c}0.883 \\
\pm \\
0.023\end{array}$ & $\begin{array}{c}3.921 \\
\pm \\
0.070\end{array}$ & $\begin{array}{c}2.043 \\
\pm \\
0.025\end{array}$ & $\begin{array}{c}1.715 \\
\pm \\
0.017\end{array}$ & $\begin{array}{c}0.923 \\
\pm \\
0.015\end{array}$ & $\begin{array}{c}1.789 \\
\pm \\
0.023\end{array}$ & $\begin{array}{c}4.090 \\
\pm \\
0.032\end{array}$ & $\begin{array}{c}4.967 \\
\pm \\
0.054\end{array}$ & $\begin{array}{c}1.211 \\
\pm \\
0.001\end{array}$ & $\begin{array}{c}0.857 \\
\pm \\
0.032\end{array}$ & $\begin{array}{c}6.177 \\
\pm \\
0.070\end{array}$ & $\begin{array}{c}8.558 \\
\pm \\
0.010\end{array}$ \\
\hline 1920 & $\begin{array}{c}0.615 \\
\pm \\
0.057\end{array}$ & $\begin{array}{c}0.217 \\
\pm \\
0.083\end{array}$ & $\begin{array}{c}6.002 \\
\pm \\
0.020\end{array}$ & $\begin{array}{c}3.567 \\
\pm \\
0.045\end{array}$ & $\begin{array}{c}1.064 \\
\pm \\
0.044\end{array}$ & $\begin{array}{c}2.077 \\
\pm \\
0.031\end{array}$ & $\begin{array}{c}3.290 \\
\pm \\
0.017\end{array}$ & $\begin{array}{c}3.881 \\
\pm \\
0.049\end{array}$ & $\begin{array}{c}0.358 \\
\pm \\
0.038\end{array}$ & $\begin{array}{c}1.215 \\
\pm \\
0.061\end{array}$ & $\begin{array}{c}7.187 \\
\pm \\
0.062\end{array}$ & PD \\
\hline
\end{tabular}

Note: PD-Plant died.

From Table 2, it can be seen that $H$. annuus, was the highest accumulator of lead in solutions, followed by $C$. bengelensis, Z. mays and finally A. hybridus. The accumulation of $\mathrm{Pb}$ in $H$. annuus was not statistically different from that of $C$. bengelensis for all the three harvests $(\mathrm{P}=0.5)$ even though $\mathrm{Pb}$ concentration in these plants was statistically different from that of Z. mays and A. hybridus. In our study, H. Annuus and $C$. bengelensis have shown a high ability to remove $\mathrm{Pb}$ from waste water effluent but the ability of $\mathrm{Z}$. Mays and $A$. Hybridus is low. These results are in agreement with findings recorded by Ghosh and Singh (2005), where several plants were investigated for their bioaccumulation of $\mathrm{Pb}$ from waste water; H. annuus was found to have the greatest ability.

\subsection{Cadmium}

Table 3 presents the range of $\mathrm{Cd}$ concentrations as (0.138-19.690), (0.082-22.367), (2.482-9.146) and (0.201-9.972) $\mathrm{g} \mathrm{kg}^{-1}$ for Z. mays, A. hybridus, H. annuus and C. bengelensis respectively. The concentration of $\mathrm{Cd}$ obtained for these treatments were greater than the value used to define $\mathrm{Cd}$ hyperaccumulation $\left(0.1 \mathrm{gkg}^{-1}\right)$. 
NATHAN, O.; NJERI, K. P.; RANG'ondi, O. E.; SARIMA, C. J. The potential of Zea mays, Commelina bengelensis, Helianthus annuus and Amaranthus hybridus for phytoremediation of waste water. Ambi-Agua, Taubaté, v. 7, n. 3, p. 51-60, 2012. (http://dx.doi.org/10.4136/ambi-agua.684)

Table 3. Concentration of cadmium $\left(\mathrm{gkg}^{-1}\right)$ in Z. mays, C. bengelensis and A. hybridus grown in contaminated soil for the first, second and third harvests over three months period.

\begin{tabular}{|c|c|c|c|c|c|c|c|c|c|c|c|c|}
\hline \multirow{2}{*}{$\begin{array}{c}\begin{array}{c}\text { Metal } \\
\text { Cd }\end{array} \\
\text { Treat. }\end{array}$} & \multicolumn{4}{|c|}{$1^{\text {st }}$ (Month) } & \multicolumn{4}{|c|}{$2^{\text {nd }}$ (Month) } & \multicolumn{4}{|c|}{$3^{\text {rd }}$ (Month) } \\
\hline & $\begin{array}{c}\text { A. } \\
\text { hybridus }\end{array}$ & $\begin{array}{c}\boldsymbol{Z} . \\
\text { mays }\end{array}$ & $\begin{array}{c}\boldsymbol{C} . \\
\text { bengelensi }\end{array}$ & $\begin{array}{c}\boldsymbol{H} . \\
\text { annuus }\end{array}$ & $\underset{\text { hybridus }}{\boldsymbol{A} .}$ & $\begin{array}{l}\boldsymbol{Z} . \\
\text { mays }\end{array}$ & $\begin{array}{c}C . \\
\text { bengelensis }\end{array}$ & $\begin{array}{c}\boldsymbol{H} . \\
\text { annuus }\end{array}$ & $\begin{array}{c}A . \\
\text { hybridus }\end{array}$ & $\begin{array}{c}\boldsymbol{Z} . \\
\text { mays }\end{array}$ & $\begin{array}{c}C . \\
\text { bengelensi }\end{array}$ & $\begin{array}{c}\boldsymbol{H} . \\
\text { annuus }\end{array}$ \\
\hline \multirow[t]{3}{*}{0} & 0.000 & 0.000 & 0.000 & 0.000 & 0.000 & 0.000 & 0.000 & 0.000 & 0.000 & 0.000 & 0.000 & 0.000 \\
\hline & \pm & \pm & \pm & \pm & \pm & \pm & \pm & \pm & \pm & \pm & \pm & \pm \\
\hline & 0.000 & 0.000 & 0.000 & 0.000 & 0.000 & 0.000 & 0.000 & 0.000 & 0.000 & 0.000 & 0.000 & 0.000 \\
\hline \multirow[t]{2}{*}{10} & $\begin{array}{c}0.163 \\
+\end{array}$ & $\begin{array}{c}0.273 \\
+\end{array}$ & $\begin{array}{c}0.474 \\
+\end{array}$ & $\begin{array}{c}2.555 \\
+\end{array}$ & $\begin{array}{c}0.082 \\
+\end{array}$ & $\begin{array}{c}0.138 \\
+\end{array}$ & $\begin{array}{c}0.201 \\
+\end{array}$ & $\begin{array}{c}2.626 \\
+\end{array}$ & $\begin{array}{c}0.206 \\
+\end{array}$ & $\begin{array}{l}0.326 \\
+0\end{array}$ & $\begin{array}{c}0.289 \\
+\end{array}$ & $\begin{array}{l}2.482 \\
+0\end{array}$ \\
\hline & 0.013 & 0.052 & 0.008 & 0.013 & 0.002 & 0.010 & 0.007 & 0.001 & 0.010 & 017 & 0.006 & .005 \\
\hline \multirow[t]{2}{*}{20} & 0.455 & 0.450 & $\begin{array}{c}0.796 \\
+\end{array}$ & $\begin{array}{c}3.266 \\
+\end{array}$ & $\begin{array}{c}0.439 \\
+\end{array}$ & $\begin{array}{c}0.774 \\
+\end{array}$ & $\begin{array}{c}1.365 \\
+\end{array}$ & 5.701 & 0.636 & $\begin{array}{c}0.751 \\
+0\end{array}$ & 1.287 & 3.159 \\
\hline & $\begin{array}{c} \pm \\
0.013\end{array}$ & $\begin{array}{c} \pm \\
0.007\end{array}$ & $\begin{array}{c} \pm \\
0.009\end{array}$ & $\begin{array}{c} \pm \\
0.011\end{array}$ & $\begin{array}{c} \pm \\
0.008\end{array}$ & $\begin{array}{c} \pm \\
0.007\end{array}$ & $\begin{array}{c} \pm \\
0.008\end{array}$ & $\begin{array}{c} \pm \\
0.030\end{array}$ & $\begin{array}{c} \pm \\
0.011\end{array}$ & $\begin{array}{l} \pm 0 \\
021\end{array}$ & $\begin{array}{c} \pm \\
0.009\end{array}$ & $\begin{array}{c} \pm \\
0.020\end{array}$ \\
\hline \multirow[t]{2}{*}{40} & $\begin{array}{c}0.683 \\
\pm\end{array}$ & $\begin{array}{c}0.602 \\
\pm\end{array}$ & $\begin{array}{l}2.129 \\
\pm\end{array}$ & $\begin{array}{l}5.321 \\
\pm\end{array}$ & $\begin{array}{l}0.435 \\
\pm\end{array}$ & $\begin{array}{c}1.005 \\
\pm\end{array}$ & $\begin{array}{l}2.121 \\
\pm\end{array}$ & $\begin{array}{c}6.414 \\
\pm 0\end{array}$ & $\begin{array}{c}0.395 \\
\pm\end{array}$ & PD & $\begin{array}{c}1.632 \\
\pm\end{array}$ & $\begin{array}{c}5.669 \\
\pm 0\end{array}$ \\
\hline & 0.012 & 0.025 & 0.010 & 0.014 & 0.012 & 0.010 & 0.011 & .007 & 0.018 & & 0.010 & .006 \\
\hline \multirow[t]{2}{*}{80} & 2.576 & 2.734 & 2.964 & 6.402 & 1.538 & 2.632 & 5.912 & 6.965 & 1.369 & PD & 3.950 & 7.046 \\
\hline & $\begin{array}{c} \pm \\
0.093\end{array}$ & $\begin{array}{c} \pm \\
0.100\end{array}$ & $\stackrel{ \pm}{ \pm}$ & $\begin{array}{c} \pm \\
.066\end{array}$ & $\begin{array}{c} \pm \\
0.003\end{array}$ & $\begin{array}{l} \pm 0 \\
.100\end{array}$ & $\stackrel{ \pm}{ \pm}$ & $\begin{array}{c} \pm \\
0.018\end{array}$ & $\stackrel{ \pm}{ \pm}$ & & $\begin{array}{c} \pm \\
0.004\end{array}$ & $\stackrel{ \pm}{ \pm}$ \\
\hline \multirow[t]{2}{*}{160} & 4.419 & 6.001 & 3.668 & 7.513 & 5.246 & 7.754 & 7.337 & 8.887 & 6.560 & PD & 6.811 & PD \\
\hline & $\begin{array}{c} \pm \\
0.014\end{array}$ & $\begin{array}{c} \pm \\
0.015\end{array}$ & $\begin{array}{c} \pm \\
0.016\end{array}$ & $\begin{array}{c} \pm \\
0.023\end{array}$ & $\begin{array}{c} \pm \\
0.084\end{array}$ & $\begin{array}{c} \pm \\
0.063\end{array}$ & $\begin{array}{c} \pm \\
0.054\end{array}$ & $\begin{array}{c} \pm \\
0.001\end{array}$ & $\begin{array}{c} \pm \\
0.056\end{array}$ & & $\begin{array}{c} \pm \\
0.009\end{array}$ & \\
\hline \multirow[t]{2}{*}{320} & 10.200 & 10.677 & 5.706 & 8.966 & 16.567 & PD & 8.874 & 9.052 & 15.807 & PD & 7.035 & PD \\
\hline & $\begin{array}{c} \pm \\
0.070\end{array}$ & $\begin{array}{c} \pm \\
0.035\end{array}$ & $\begin{array}{c} \pm \\
0.048\end{array}$ & $\begin{array}{c} \pm \\
0.010\end{array}$ & $\begin{array}{c} \pm \\
0.049\end{array}$ & & $\stackrel{ \pm}{ \pm}$ & $\begin{array}{c} \pm \\
0.006\end{array}$ & $\begin{array}{c} \pm \\
0.119\end{array}$ & & $\begin{array}{c} \pm \\
0.005\end{array}$ & \\
\hline \multirow[t]{2}{*}{640} & 19.233 & 19.690 & 5.901 & 9.132 & 22.367 & PD & 9.972 & 9.146 & PD & PD & 8.729 & PD \\
\hline & $\begin{array}{c} \pm \\
0.179\end{array}$ & $\stackrel{ \pm}{ \pm}$ & $\begin{array}{c} \pm \\
0.019\end{array}$ & $\begin{array}{c} \pm \\
0.004\end{array}$ & $\stackrel{ \pm}{ \pm}$ & & $\begin{array}{c} \pm \\
0.020\end{array}$ & $\begin{array}{c} \pm \\
0.004\end{array}$ & & & $\begin{array}{c} \pm \\
0.007\end{array}$ & \\
\hline
\end{tabular}

Note: PD-Plant died.

In a research done by Antonkiewicz and Jasiewicz (2002) in contaminated soils, the Cd content in Z. mays and A. hybridus was $0.000-0.070 \mathrm{~g} \mathrm{~kg}^{-1}$ and $0.001-0.060 \mathrm{~g} \mathrm{~kg}^{-1}$ respectively while in another study by Lombi et al. (2001), a range of $0.60-0.576 \mathrm{~g} \mathrm{~kg}^{-1}$ was obtained for T. caerulescens. The highest value of Cd concentration of $1.800 \mathrm{~g} \mathrm{~kg}^{-1}$ was recorded in leaves of T. caerulescens in a research carried out by Baker and Walker (1990). The values obtained in the current study are higher than those recorded in these studies. Cd concentrations above $20 \mathrm{mg} / \mathrm{l}, 80 \mathrm{mg} / \mathrm{l}$ and $320 \mathrm{mg} / \mathrm{l}$ were found to be toxic for the growth of Z. mays, H. annuus and $A$. hybridus respectively. $C$. bengelensis did not show any toxicity signs to $\mathrm{Cd}$ which gives the plant an added advantage over the other plants in phytoremediation of wastewater containing higher concentrations of $\mathrm{Cd}$.

From table 3, the high uptake of $\mathrm{Cd}$ by $H$. annuus is comparable to that of $C$. bengelensis while the uptake of $\mathrm{Cd}$ by $Z$. mays is lower than that of A. hybridus. Similar trend was observed by Antonkiewicz and Jasiewicz (2002), where the Cd content in the top parts of $A$. hybridus was found to be higher than that of Z. mays.

$H$. annuus has the highest removal of $\mathrm{Cd}$ from solutions, followed by $C$. bengelensis, then A. hybridus and finally Z. mays.

\subsection{Copper}

Table 4 presents the range of copper concentration $(0.213-35.770) \mathrm{g} \mathrm{kg}^{-1}$ for Z. mays, (0.230-43.273) $\mathrm{g} \mathrm{kg}^{-1}$ for A. hybridus, (1.786-15.995) $\mathrm{g} \mathrm{kg}^{-1}$ for H. annuus and (0.514- 
NATHAN, O.; NJERI, K. P.; RANG'ondi, O. E.; SARIMA, C. J. The potential of Zea mays, Commelina bengelensis, Helianthus annuus and Amaranthus hybridus for phytoremediation of waste water. Ambi-Agua, Taubaté, v. 7, n. 3, p. 51-60, 2012. (http://dx.doi.org/10.4136/ambi-agua.684)

20.807) $\mathrm{g} \mathrm{kg}^{-1}$ for $C$. bengelensis. These values are higher than those obtained in earlier studies where the range of $\mathrm{Cu}$ values indicated as $0.001-0.0075 \mathrm{~g} \mathrm{~kg}^{-1}$ in aquatic macrophytes (Espinoza-Quiñones et al., 2005), $0.0015-0.0189 \mathrm{~g} \mathrm{~kg}^{-1}$ in $Z$. mays and $0.085-0.0327 \mathrm{~g} \mathrm{~kg}^{-1}$ in H. annuus (Podar et al., 2004) and 0.0413- $0.806 \mathrm{~g} \mathrm{~kg}^{-1}$ in Z. mays roots with EDTA (Lombi et al., 2001). The values obtained for all the four plants at treatments of 640 and $1280 \mathrm{ppm}$ are above the value of a $\mathrm{Cu}$ hyperacumulator, Ipomea alpina of $12.300 \mathrm{~g} \mathrm{~kg}^{-1}$ (Baker and Walker, 1990). However the plants were found to suffer from $\mathrm{Cu}$ phytotoxicity at $\mathrm{Cu}$ concentrations higher than $80 \mathrm{ppm}$. This implies that although the plants can be used for phytoremediation of wastewater contaminated with $\mathrm{Cu}$, their remediation potential would be limited by phytotoxicity of $\mathrm{Cu}$. It was also noted that in the uptake of $\mathrm{Cu}$ by $C$. bengelensis, the initial ( $1^{\text {st }}$ harvest), was not as high as in the other plants and this is probably why it was possible to obtain the $2^{\text {nd }}$ harvest for all treatments.

Table 4: Concentration of copper $\left(\mathrm{gkg}^{-1}\right)$ in Z. mays, C. bengelensis and A. hybridus grown in contaminated soil for the first, second and third harvests over three months period.

\begin{tabular}{|c|c|c|c|c|c|c|c|c|c|c|c|c|}
\hline \multirow{2}{*}{$\begin{array}{l}\begin{array}{l}\text { Metal } \\
\text { Cu }\end{array} \\
\text { Treat. }\end{array}$} & \multicolumn{4}{|c|}{$1^{\text {st }}$ (Month) } & \multicolumn{4}{|c|}{$2^{\text {nd }}$ (Month) } & \multicolumn{4}{|c|}{$3^{\text {rd }}$ (Month) } \\
\hline & $\begin{array}{c}\text { A. } \\
\text { hvbridus }\end{array}$ & $\begin{array}{c}Z . \\
\text { mavs }\end{array}$ & $\begin{array}{c}\boldsymbol{C} . \\
\text { bengelensis } \\
\end{array}$ & $\begin{array}{c}\boldsymbol{H} . \\
\text { annuus }\end{array}$ & $\begin{array}{c}\text { A. } \\
\text { hvbridus }\end{array}$ & $\begin{array}{c}Z . \\
\text { mavs }\end{array}$ & $\begin{array}{c}\boldsymbol{C} . \\
\text { bengelensis }\end{array}$ & $\begin{array}{c}\boldsymbol{H} . \\
\text { aпnuи }\end{array}$ & $\begin{array}{c}\text { A. } \\
\text { hvbridus }\end{array}$ & $\begin{array}{c}Z . \\
\text { mavs }\end{array}$ & $\begin{array}{c}C . \\
\text { bengelensis }\end{array}$ & $\begin{array}{c}\boldsymbol{H} . \\
\text { annuиs }\end{array}$ \\
\hline 0 & $\begin{array}{c}0.000 \\
\pm \\
0.000\end{array}$ & $\begin{array}{c}0.000 \\
\pm \\
0.000\end{array}$ & $\begin{array}{c}0.000 \\
\pm \\
0.000\end{array}$ & $\begin{array}{c}0.000 \\
\pm \\
0.000\end{array}$ & $\begin{array}{c}0.000 \\
\pm \\
0.000\end{array}$ & $\begin{array}{c}0.000 \\
\pm \\
0.000\end{array}$ & $\begin{array}{c}0.000 \\
\pm \\
0.000\end{array}$ & $\begin{array}{c}0.000 \\
\pm \\
0.000\end{array}$ & $\begin{array}{c}0.000 \\
\pm \\
0.000\end{array}$ & $\begin{array}{c}0.000 \\
\pm \\
0.000\end{array}$ & $\begin{array}{c}0.000 \\
\pm \\
0.000\end{array}$ & $\begin{array}{c}0.000 \\
\pm \\
0.000\end{array}$ \\
\hline 20 & $\begin{array}{c}0.230 \\
\pm \\
0.016\end{array}$ & $\begin{array}{c}0.493 \\
\pm \\
0.026\end{array}$ & $\begin{array}{c}0.514 \\
\pm \\
0.009\end{array}$ & $\begin{array}{c}1.786 \\
\pm \\
0.018\end{array}$ & $\begin{array}{c}0.257 \\
\pm \\
0.043\end{array}$ & $\begin{array}{c}0.213 \\
\pm \\
0.047\end{array}$ & $\begin{array}{c}0.699 \\
\pm \\
0.023\end{array}$ & $\begin{array}{c}2.099 \\
\pm \\
0.013\end{array}$ & $\begin{array}{c}0.319 \\
\pm \\
0.009\end{array}$ & $\begin{array}{c}0.371 \\
\pm \\
0.019\end{array}$ & $\begin{array}{c}0.839 \\
\pm \\
0.013\end{array}$ & $\begin{array}{c}2.987 \\
\pm \\
0.020\end{array}$ \\
\hline 40 & $\begin{array}{c}0.596 \\
\pm \\
0.017\end{array}$ & $\begin{array}{c}1.389 \\
\pm \\
0.024\end{array}$ & $\begin{array}{c}1.548 \\
\pm \\
0.009\end{array}$ & $\begin{array}{c}2.664 \\
\pm \\
0.016\end{array}$ & $\begin{array}{c}0.333 \\
\pm \\
0.014\end{array}$ & $\begin{array}{c}1.943 \\
\pm \\
0.119\end{array}$ & $\begin{array}{c}3.425 \\
\pm \\
0.024\end{array}$ & $\begin{array}{c}4.278 \\
\pm \\
0.026\end{array}$ & $\begin{array}{c}0.697 \\
\pm \\
0.020\end{array}$ & $\begin{array}{c}0.866 \\
\pm \\
0.024\end{array}$ & $\begin{array}{c}1.115 \\
\pm \\
0.020\end{array}$ & $\begin{array}{c}3.476 \\
\pm \\
0.022\end{array}$ \\
\hline 80 & $\begin{array}{c}1.900 \\
\pm \\
0.022\end{array}$ & $\begin{array}{c}2.384 \\
\pm \\
0.265\end{array}$ & $\begin{array}{c}2.150 \\
\pm \\
0.023\end{array}$ & $\begin{array}{c}4.502 \\
\pm \\
0.053\end{array}$ & $\begin{array}{c}3.019 \\
\pm \\
0.0136\end{array}$ & $\begin{array}{c}3.504 \\
\pm \\
0.042\end{array}$ & $\begin{array}{c}5.553 \\
\pm \\
0.012\end{array}$ & $\begin{array}{c}6.647 \\
\pm \\
0.007\end{array}$ & $\begin{array}{c}0.958 \\
\pm \\
0.016\end{array}$ & $\begin{array}{c}3.087 \\
\pm \\
0.049\end{array}$ & $\begin{array}{c}6.675 \\
\pm \\
0.013\end{array}$ & PD \\
\hline 160 & $\begin{array}{c}1.918 \\
\pm \\
0.069\end{array}$ & $\begin{array}{c}3.325 \\
\pm \\
0.060\end{array}$ & $\begin{array}{c}2.958 \\
\pm \\
0.027\end{array}$ & $\begin{array}{c}5.851 \\
\pm \\
0.018\end{array}$ & $\begin{array}{c}5.486 \\
\pm \\
0.099\end{array}$ & $\begin{array}{c}4.347 \\
\pm \\
0.346\end{array}$ & $\begin{array}{c}5.876 \\
\pm \\
0.038\end{array}$ & $\begin{array}{c}8.308 \\
\pm \\
0.047\end{array}$ & $\begin{array}{c}1.858 \\
\pm \\
0.028\end{array}$ & PD & PD & PD \\
\hline 320 & $\begin{array}{c}6.072 \\
\pm \\
0.023\end{array}$ & $\begin{array}{c}8.080 \\
\pm \\
0.147\end{array}$ & $\begin{array}{c}5.122 \\
\pm \\
0.126\end{array}$ & $\begin{array}{c}7.025 \\
\pm \\
0.028\end{array}$ & $\begin{array}{c}9.966 \\
\pm \\
0.166\end{array}$ & PD & $\begin{array}{c}12.115 \\
\pm \\
0.074\end{array}$ & $\begin{array}{c}10.782 \\
\pm \\
0.068\end{array}$ & PD & PD & PD & PD \\
\hline 640 & $\begin{array}{c}26.753 \\
\pm \\
0.121\end{array}$ & $\begin{array}{c}24.013 \\
\pm \\
0.085\end{array}$ & $\begin{array}{c}6.581 \\
\pm \\
0.016\end{array}$ & $\begin{array}{c}12.875 \\
\pm \\
0.006\end{array}$ & PD & PD & $\begin{array}{c}11.697 \\
\pm \\
0.049\end{array}$ & PD & $\mathrm{PD}$ & PD & PD & PD \\
\hline 1280 & $\begin{array}{c}43.273 \\
\pm \\
0.145\end{array}$ & $\begin{array}{c}35.770 \\
\pm \\
0.185\end{array}$ & $\begin{array}{c}7.960 \\
\pm \\
0.052\end{array}$ & $\begin{array}{c}15.995 \\
\pm \\
0.031\end{array}$ & PD & PD & $\begin{array}{c}20.807 \\
\pm \\
0.147\end{array}$ & PD & PD & PD & PD & PD \\
\hline
\end{tabular}

From Table 4, the $\mathrm{Cu}$ concentration in H. annuus, C. bengelensis, Z. mays and $A$. hybridus was found to increase as the concentration in the solution increased. The first and second harvests did not show much difference in their accumulation for the four plants and by third harvest, most of the plants had died in solutions containing high levels of $\mathrm{Cu}$ contamination. In phytoremediation, due to $\mathrm{Cu}$ phytotoxicity at high concentrations, the plants should be removed from the contaminated aqueous solutions on the $4^{\text {th }}$ day.

Comparison of the uptake of $\mathrm{Cu}$ by the four plants at the end of the first four days reveals that at $\mathrm{Cu}$ concentrations below $640 \mathrm{mg} \mathrm{L}^{-1}, H$. annuus has the highest uptake of $\mathrm{Cu}$, while at concentrations of 640 and $1280 \mathrm{mg} \mathrm{L}^{-1}$, Z. mays and A. hybridus have the highest uptake. 
NATHAN, O.; NJERI, K. P.; RANG'ondi, O. E.; SARIMA, C. J. The potential of Zea mays, Commelina bengelensis, Helianthus annuus and Amaranthus hybridus for phytoremediation of waste water. Ambi-Agua, Taubaté, v. 7, n. 3, p. 51-60, 2012. (http://dx.doi.org/10.4136/ambi-agua.684)

\subsection{Zinc}

Table 5 presents the $\mathrm{Zn}$ concentration in Z. mays, A. hybridus, H. annuus and $C$. bengelensis were found to be in the range (2.282-8.307), (1.141-7.593), (2.674-6.992) and (1.876-7.770) $\mathrm{g} \mathrm{kg}^{-1}$, respectively. These values are lower than the value used to define a $\mathrm{Zn}$ hyper accumulator $\left(10.0 \mathrm{~g} \mathrm{~kg}^{-1}\right)$, (Lasat, 2000), and much lower than a value of $38.055 \mathrm{~g} \mathrm{~kg}^{-1}$ obtained by Hinchman et al. (1996) in the roots of hybrid poplar. The values are comparable to those obtained by Lombi et al. (2001) and Gremion et al. (2004) that is $1.868-9.187 \mathrm{~g} \mathrm{~kg}^{-1}$ and $1.600-10.000 \mathrm{~g} \mathrm{~kg}^{-1}$ respectively for $T$. caerulescens which is a documented $\mathrm{Zn}$ hyperaccumulator. However, the values are lower than the greatest $\mathrm{Zn}$ uptake of $39.600 \mathrm{~g} \mathrm{~kg}^{-1}$ in $T$. caerulescens leaves. This means that the $\mathrm{Zn}$ uptake by these plants is quite significant though they cannot be said to be hyperaccumulative in solutions.

Table 5: Concentration of zinc $\left(\mathrm{g} \mathrm{kg}^{-1}\right)$ in $Z$. mays, C. bengelensis and A. hybridus grown in contaminated soil for the first, second and third harvests over three months period.

\begin{tabular}{|c|c|c|c|c|c|c|c|c|c|c|c|c|}
\hline \multirow{2}{*}{$\begin{array}{c}\begin{array}{c}\text { Metal } \\
\text { Zn }\end{array} \\
\text { Treat. }\end{array}$} & \multicolumn{4}{|c|}{$\mathbf{1}^{\text {st }}$ (Month) } & \multicolumn{4}{|c|}{$2^{\text {nd }}$ (Month) } & \multicolumn{4}{|c|}{$3^{\text {rd }}$ (Month) } \\
\hline & $\begin{array}{c}\text { A. } \\
\text { hybridus }\end{array}$ & $\begin{array}{c}\boldsymbol{Z} . \\
\text { mays }\end{array}$ & $\begin{array}{c}\boldsymbol{C} . \\
\text { bengelensis }\end{array}$ & $\begin{array}{c}\boldsymbol{H} . \\
\text { апnиus }\end{array}$ & $\begin{array}{c}\text { A. } \\
\text { hybridus }\end{array}$ & $\begin{array}{l}\boldsymbol{Z} . \\
\text { mays }\end{array}$ & $\begin{array}{c}\boldsymbol{C} . \\
\text { bengelensis }\end{array}$ & $\begin{array}{c}\boldsymbol{H} . \\
\text { annuus }\end{array}$ & $\begin{array}{c}\text { A. } \\
\text { hybridus }\end{array}$ & $\underset{\text { mays }}{\mathbf{Z}}$ & $\underset{\text { bengelensis }}{\boldsymbol{C} .}$ & $\begin{array}{c}\boldsymbol{H} . \\
\text { апnuиs }\end{array}$ \\
\hline 0 & $\begin{array}{c}0.00 \\
\pm \\
0.00\end{array}$ & $\begin{array}{c}0.00 \\
\pm \\
0.00\end{array}$ & $\begin{array}{c}0.00 \\
\pm \\
0.00\end{array}$ & $\begin{array}{c}0.00 \\
\pm \\
0.00\end{array}$ & $\begin{array}{c}0.000 \\
\pm \\
0.000\end{array}$ & $\begin{array}{c}0.00 \\
\pm \\
0.000\end{array}$ & $\begin{array}{c}0.000 \\
\pm \\
0.000\end{array}$ & $\begin{array}{c}0.000 \\
\pm \\
0.000\end{array}$ & $\begin{array}{c}0.000 \\
\pm \\
0.000\end{array}$ & $\begin{array}{c}0.000 \\
\pm \\
0.000\end{array}$ & $\begin{array}{c}0.000 \\
\pm \\
0.000\end{array}$ & $\begin{array}{c}0.000 \\
\pm \\
0.000\end{array}$ \\
\hline 50 & $\begin{array}{c}1.141 \\
\pm \\
0.006\end{array}$ & $\begin{array}{c}2.417 \\
\pm \\
0.007\end{array}$ & $\begin{array}{c}2.303 \\
\pm \\
0.007\end{array}$ & $\begin{array}{c}2.674 \\
\pm \\
0.020\end{array}$ & $\begin{array}{c}1.327 \\
\pm \\
0.007\end{array}$ & $\begin{array}{c}2.282 \\
\pm \\
0.007\end{array}$ & $\begin{array}{c}1.876 \\
\pm \\
0.005\end{array}$ & $\begin{array}{c}4.456 \\
\pm \\
0.007\end{array}$ & $\begin{array}{c}1.743 \\
\pm \\
0.021\end{array}$ & $\begin{array}{c}3.084 \\
\pm \\
0.016\end{array}$ & $\begin{array}{c}2.014 \\
\pm \\
0.006\end{array}$ & $\begin{array}{c}4.301 \\
\pm \\
0.024\end{array}$ \\
\hline 100 & $\begin{array}{c}1.398 \\
\pm \\
0.002\end{array}$ & $\begin{array}{c}4.246 \\
\pm \\
0.007\end{array}$ & $\begin{array}{c}3.134 \\
\pm \\
0.011\end{array}$ & $\begin{array}{c}4.520 \\
\pm \\
0.012\end{array}$ & $\begin{array}{c}5.293 \\
\pm \\
0.002\end{array}$ & $\begin{array}{c}4.679 \\
\pm \\
0.013\end{array}$ & $\begin{array}{c}4.390 \\
\pm \\
0.051\end{array}$ & $\begin{array}{c}5.552 \\
\pm \\
0.003\end{array}$ & $\begin{array}{c}4.455 \\
\pm \\
0.011\end{array}$ & $\begin{array}{c}4.912 \\
\pm \\
0.013\end{array}$ & $\begin{array}{c}3.056 \\
\pm \\
0.008\end{array}$ & $\begin{array}{c}5.251 \\
\pm \\
0.004\end{array}$ \\
\hline 200 & $\begin{array}{c}3.943 \\
\pm \\
0.006\end{array}$ & $\begin{array}{c}5.967 \\
\pm \\
0.004\end{array}$ & $\begin{array}{c}3.491 \\
\pm \\
0.009\end{array}$ & $\begin{array}{c}5.921 \\
\pm \\
0.027\end{array}$ & $\begin{array}{c}6.181 \\
\pm \\
0.012\end{array}$ & $\begin{array}{c}6.456 \\
\pm \\
0.006\end{array}$ & $\begin{array}{c}5.306 \\
\pm \\
0.024\end{array}$ & $\begin{array}{c}6.141 \\
\pm \\
0.005\end{array}$ & $\begin{array}{c}6.662 \\
\pm \\
0.006\end{array}$ & $\begin{array}{c}6.447 \\
\pm \\
0.014\end{array}$ & $\begin{array}{c}3.22 \\
1 \pm \\
0.009\end{array}$ & $\begin{array}{c}5.704 \\
\pm \\
0.006\end{array}$ \\
\hline 400 & $\begin{array}{c}5.734 \\
\pm \\
0.009\end{array}$ & $\begin{array}{c}6.572 \\
\pm \\
0.007\end{array}$ & $\begin{array}{c}5.170 \\
\pm \\
0.029\end{array}$ & $\begin{array}{c}5.740 \\
\pm \\
0.028\end{array}$ & $\begin{array}{c}6.087 \\
\pm \\
0.007\end{array}$ & $\begin{array}{c}6.813 \\
\pm \\
0.014\end{array}$ & $\begin{array}{c}6.367 \\
\pm \\
0.012\end{array}$ & $\begin{array}{c}6.353 \\
\pm \\
0.075\end{array}$ & $\begin{array}{c}6.509 \\
\pm \\
0.007\end{array}$ & $\begin{array}{c}7.981 \\
\pm \\
0.012\end{array}$ & $\begin{array}{c}7.148 \\
\pm \\
0.004\end{array}$ & PD \\
\hline 800 & $\begin{array}{c}7.114 \\
\pm \\
0.003\end{array}$ & $\begin{array}{c}7.263 \\
\pm \\
0.003\end{array}$ & $\begin{array}{c}5.469 \\
\pm \\
0.027\end{array}$ & $\begin{array}{c}6.137 \\
\pm \\
0.009\end{array}$ & $\begin{array}{c}7.188 \\
\pm \\
0.013\end{array}$ & PD & $\begin{array}{c}7.272 \\
\pm \\
0.012\end{array}$ & $\begin{array}{c}5.986 \\
\pm \\
0.001\end{array}$ & PD & PD & $\begin{array}{c}7.489 \\
\pm \\
0.014\end{array}$ & PD \\
\hline 1600 & $\begin{array}{c}7.730 \\
\pm \\
0.009\end{array}$ & $\begin{array}{c}7.813 \\
\pm \\
0.006\end{array}$ & $\begin{array}{c}6.496 \\
\pm \\
0.021\end{array}$ & $\begin{array}{c}6.559 \\
\pm \\
0.117\end{array}$ & PD & PD & $\begin{array}{c}7.410 \\
\pm \\
0.014\end{array}$ & $\begin{array}{c}6.602 \\
\pm \\
0.011\end{array}$ & PD & PD & PD & PD \\
\hline 3200 & $\begin{array}{c}7.593 \\
\pm \\
0.010\end{array}$ & $\begin{array}{c}8.307 \\
\pm \\
0.010\end{array}$ & $\begin{array}{c}7.121 \\
\pm \\
0.049\end{array}$ & $\begin{array}{c}6.861 \\
\pm \\
0.014\end{array}$ & PD & PD & $\begin{array}{c}7.770 \\
\pm \\
0.003\end{array}$ & $\begin{array}{c}6.992 \\
\pm \\
0.015\end{array}$ & PD & PD & PD & PD \\
\hline
\end{tabular}

Table 5 presents the Zn concentration in Z. mays, A. hybridus, H. annuus and $C$. bengelensis with increase in the metal concentration in solution. The uptake of $\mathrm{Zn}$ by the four plants for the $1^{\text {st }}$ harvest (first 4 days) did not show much difference in concentration.

$H$. annuus and $C$. bengelensis were found to be more tolerant to high $\mathrm{Zn}$ concentration since it was possible to obtain data for all the 8 treatments up to the second harvest ( $8^{\text {th }}$ day) while at high concentration Z. mays and A. hybridus died off by first harvest ( $4^{\text {th }}$ day). Due to their tolerance of high levels of $\mathrm{Zn}$ and high uptake, $C$. bengelensis and $H$. annuus would be the best out of the four plants to be used to remove zinc from solutions contaminated with zinc. 


\section{CONCLUSIONS}

In this study it was found out that the heavy metal uptake by the four plants analyzed was different regarding the metal contamination. In solutions, the uptake of metals by plants may be ranked from the highest to the lowest in the following order:

Lead: $\quad$ Helianthus, Commelina, Zea mays, Amaranthus;

Cadmium: Helianthus, Commelina, Amaranthus, Zea mays;

Copper: Helianthus, Commelina, Zea mays, Amaranthus;

Zinc: $\quad$ Helianthus, Zea mays, Amaranthus, Commelina;

Helianthus, Commelina and Zea mays have shown a high ability to remove $\mathrm{Pb}$ from waste water.

The comparison of individual elements indicates much higher accumulation of $\mathrm{Cd}, \mathrm{Cu}$, and $\mathrm{Zn}$ in the plants than that of $\mathrm{Pb}$.

Helianthus, Commelina, Zea mays and Amaranthus have been found to take up a lot of these heavy metals especially $\mathrm{Zn}, \mathrm{Cd}$ and $\mathrm{Cu}$ as compared to $\mathrm{Pb}$, hence these plants can be used for phytoremediation of these heavy metals at moderate concentration. These can be effectively done if the levels of concentration are below 960, 800, 40 and $20 \mathrm{mg} \mathrm{L}^{-1}$ of solution of $\mathrm{Pb}, \mathrm{Zn}, \mathrm{Cu}$ and $\mathrm{Cd}$, respectively. From the current study it was noted that the period of planting does not really matter since the concentrations of the heavy metals after 4 , 8 and 12 days were not significantly different.

\section{ACKNOWLEDGMENT}

Sincere appreciation goes to Jomo Kenyatta University for all the financial support given in the course of this study, to the technical staff of the Chemistry Department for their help in the analytical work.

\section{REFERENCES}

AJMAL, M.; MOHAMMED, A.; YOUSUF, R.; AHMAD, A. Adsorption behavior of cadmium, zinc, nickel and lead from aqueous solutions by Mangifera Indica seed shell. Indian Journal Environmental Health, v. 40, n. 1, p. 15-26, 1998.

ANTONKIEWICZ, J.; JASIEWICZ, C. The use of plants accumulating heavy metals for detoxification of chemically polluted soils. Electronic Journal of Polish Agricultural Universities, v. 5, n. 1, 2002.

BAKER, A. J. M.; WALKER, P. L. Ecophysiology of metal uptake by tolerant plants. In: SHOW, A. J. (Ed.). Heavy metal tolerance in plants: evolutionary aspects. Boca Raton: CRC Press, 1990. p. 155-157.

BAKKALOGLU, I.; BUTTER, T. J.; EVISON, L. M.; HOLLAND, F. S.; HANCOCKTT, I. C. Screening of various types biomass for removal and recovery of heavy metals ( $\mathrm{Zn}$, $\mathrm{Cu}, \mathrm{Cd})$ by biosorption, sedimentation and desorption. Water Science technology, v. 38, n. 6, p. 269-277, 1998. http://dx.doi.org/10.1016/S0273-1223(98)00587-3

CARLSON, R. W.; BAZZAZ, F. A.; ROLFE, G. L. The effect of heavy metal on plants. Environmental Pollution, v. 7, p. 241-246, 1975. 
NATHAN, O.; NJERI, K. P.; RANG'ondi, O. E.; SARIMA, C. J. The potential of Zea mays, Commelina bengelensis, Helianthus annuus and Amaranthus hybridus for phytoremediation of waste water. Ambi-Agua, Taubaté, v. 7, n. 3, p. 51-60, 2012. (http://dx.doi.org/10.4136/ambi-agua.684)

CUNNINGHAM, S. D.; SHANN, J. R.; CROWLEY, D.; ANDERSON, T. A. In: KRUEGER, E. L.; ANDERSON, T. A.; COATS, J. P. (Ed.). Phytoremediation of soil and water contaminants. Washington, DC: American Chemical Society, 1997.

CHAUDHRY, T. M.; HAYES, W. J.; KHAN, A. G.; KHOO, C. S. Phytoremediation : focusing on accumulator plants that remediate metal-contaminated soils. Australasian Journal of Ecotoxicology, v 4, p. 37-51, 1998.

DUSHENKOV, V.; NANDA KUMAR, P. B. A.; MOTTO, H.; RASKIN, I. Rhizofiltration: the use of plants to remove heavy metals from aqueous streams. Environmental Science \& Technology, v. 29, n. 5, 1239 - 1245, 1995. http://dx.doi.org/10.1021/es00005a015

ESPINOZA-QUIÑONES, F. R.; ZACARKIM, C. M.; PALACIO, S. M.; OBREGON, C. L.; ZENATTI, D. C.; GALANTE, R. M. et al. Removal of heavy metal from polluted river water using aquatic macrophytes Salvinia sp. Brazilian Journal of Physics, v. 55, n. 3b, p.744 -746, 2005. http://dx.doi.org/10.1590/S0103-97332005000500005

GHOSH, M.; SINGH. S. P. A review on phytoremediation of heavy metals and utilization of its byproducts. Applied Ecology and Environmental Research, v. 3, n. 1, p. 1-18, 2005.

GREMION, F.; CHATZINOTAS, A.; KAUFMANN, K.; VON SIGLER, W.; HARMS, H. Impacts of heavy metal contamination and phytoremediation on a microbial community during a twelve-month microcosm experiment. FEMS Microbiology Ecology, v. 48, n. 2, p. 273-283, 2004. http://dx.doi.org/10.1016/j.femsec.2004.02.004

HINCHMAN, R. R.; NEGRI, M. C.; GATLIFF, E. G. Phytoremediation: using green plants to clean up contaminated soil, groundwater and wastewater. In: INTERNATIONAL TOPICAL MEETINGS ON NUCLEAR AND HAZARDOUS WASTE MANAGEMENT, 1996, Seattle. Proceedings... Seattle: American Nuclear Society, 1996.

LASAT, M. M. The use of plants for the removal of toxic metals from contaminated soil. [S.1.]: American Association for the Advancement of Science Environmental Science and Engineering Fellow, 2000.

LOMBI, E.; ZHAO F. J.; DUNHAM, S. J.; MCGRATH, S. P. Phytoremediation of heavy metal-contaminated soils. Journal Environmental Quality, v. 30, n. 6, p. 1919- 1926, 2001. http://dx.doi.org/10.2134/jeq2001.1919

LOW, K. S.; LEE, C. K.; LIEW, S. C. Sorption of cadmium and Lead from aqueous solution by spent grain. Process Biochemistry, v. 36, n. 1/2, p. 59-64, 2000. http://dx.doi.org/10.1016/S0032-9592(00)00177-1

PODAR, D.; DOBROTA, C.; TRIFU, M. Uptake of heavy metals by maize (Zea mays) plants cultivated on mine spoils. Studia Universitatis Babes-Bolyai, Studia Biologia, v. 49, n. 1, p. 45-60, 2004a.

PODAR, D.; RAMSEY M. H.; HUTCHINGS, M. J. Effect of cadmium, zinc and substrate heterogeneity on yield, shoot metal concentration and metal uptake by Brassica juncea: implications for human risk assessment and phytoremediation. New Phytologist, v. 163, n. 2, p. 313-324, 2004b. http://dx.doi.org/10.1111/j.1469-8137.2004.01122.x

RASKIN, I.; ENSLEY, B. D. Phytoremediation of toxic metals: using plants to clean up the environment. New york: John Wiley \& Sons, 2000. p. 53-70. 
NATHAN, O.; NJERI, K. P.; RANG'ondi, O. E.; SARIMA, C. J. The potential of Zea mays, Commelina bengelensis, Helianthus annuus and Amaranthus hybridus for phytoremediation of waste water. Ambi-Agua, Taubaté, v. 7, n. 3, p. 51-60, 2012. (http://dx.doi.org/10.4136/ambi-agua.684)

REEVES, R. D.; BAKER, A. J. M. Metal accumulating plants. In: RASKIN, I.; ENSLEY, B. D. (Ed.). Phytoremediation of toxic metals: using plants to clean up the environment, 2000. p. 193-229.

SALT, E. D.; PICKERING, I. J.; PRINCE, R. C.; GLEBA, D.; DUSHENKOV, S.; SMITH, R. D. et al. Metal accumulation by aquacultured seedlings of Indian mustard. Environmental Science \& Technology, v. 31, n. 6, p. 1636-1644, 1997.

STOLTZ, E.; GREGER, M. Accumulation properties of $\mathrm{As}, \mathrm{Cd}, \mathrm{Cu}, \mathrm{Pb}$ and $\mathrm{Zn}$ by four wetland plant species growing on submerged mine tailings. Environmental and Experimental Botany, v. 47, n. 3, p. 271-280, 2002. http://dx.doi.org/10.1016/S00988472(02)00002-3

VALDMAN, E.; ERIJMAN, L.; PESSOA, F. L. P.; LEITE, S. G. F. Continuous biosorption of $\mathrm{Cu}$ and $\mathrm{Zn}$ by immobilized waste biomass Sargassum sp. Process Biochemistry, v. 36, n. 8/9, p. 869-873, 2001. http://dx.doi.org/10.1016/S0032-9592(00)00288-0

VYSLOUZILOVA, M.; TLUSTOS, P.; SZAKOVA, J.; PAVLIKOVA, D. As, Cd, Pb and Zn uptake by Salix. spp. clones grown in soils enriched by high loads of these elements. Plant, Soil and Environment, v. 49, n. 5, p. 191-196, 2003. 